\title{
Illumination effects on electrical characteristics of GaN/AIGaN/GaN heterostructures and heterostructure field effect transistors and their elimination by proper surface passivation
}

\author{
M. Fagerlind ${ }^{\mathrm{a})}$ and N. Rorsman \\ Department of Microtechnology and Nanoscience, Chalmers University of Technology, \\ SE-41296 Göteborg, Sweden
}

(Received 31 January 2012; accepted 26 May 2012; published online 12 July 2012)

\begin{abstract}
The effect of ambient illumination is investigated for differently processed GaN/AlGaN/GaN heterostructure materials. For samples of the same material with different passivation, the difference in sheet resistance of illuminated and non-illuminated material can be as large as $130 \%$ (for annealed heterostructure without passivation) and as small as 3\% (for heterostructure passivated with low pressure chemical vapor deposition (LPCVD) silicon nitride). The time constant for the decay of the persistent photoconductance (PPC) is also very different for the differently processed samples. The majority of the effect on the conductance is from photons with energies between 3.1 and $3.7 \mathrm{eV}$. The investigation indicates that delayed recombination of electrons emitted from surface states and from deep level states in the AlGaN layer dominates the PPC. A theory is formulated by which the difference in illumination sensitivity for the differently passivated materials can be explained by different distributions of electrons between the channel two dimensional electron gas and an accumulation layer formed in the cap layer. For practical heterostructure field effect transistor (HFET) measurements, the illumination sensitivity is generally lower than that of the Hall measurements. Furthermore, HFETs fabricated with the LPCVD silicon nitride passivation are practically illumination invariant. (C) 2012 American Institute of Physics. [http://dx.doi.org/10.1063/1.4730782]
\end{abstract}

\section{INTRODUCTION}

The AlGaN/GaN based heterostructure field effect transistor (HFET) technology is reaching maturity. The device concept has been proven and the effort is now more focused on improving the reliability of the transistor. In addition to excellent electrical properties, III-nitrides have bandgaps suitable for generation of photons in the visible and ultraviolet spectrum. ${ }^{1}$ The reverse process, i.e., that the III-nitrides absorb photons, is utilized in GaN-based photo-detectors. ${ }^{2}$ From an HFET perspective, photo-generation and other illumination effects are unwanted effects. For instance, the transport characteristic of the $\mathrm{AlGaN} / \mathrm{GaN}$ heterostructure is affected by persistent photoconductivity (PPC). The PPC is seen as a successive material resistivity increase when illumination of the material is suspended..$^{3-7}$ Some earlier reports on electrical effects of illumination on HFETs are as follows: Klein et al. ${ }^{8}$ use photo-ionization experiments to identify that traps in the GaN buffer are responsible for current collapse; Mizutani et al. ${ }^{9}$ use spatially resolved illumination to investigate the location of traps responsible for dispersion in HFETs; Valizadeh et al. ${ }^{10}$ investigate the effect of thermal annealing and UV illumination; Yang et al. ${ }^{11}$ use a large signal network analyzer and a spectrometer in order to investigate the effect of deep-level traps on HFET characteristics. However, in most published AlGaN/GaN HFET papers, the effect of illumination is not discussed.

\footnotetext{
${ }^{a)}$ Electronic mail: martin.fagerlind@chalmers.se.
}

The purpose of this investigation is threefold. First to quantify the illumination effect on measurements at different stages of the HFET fabrication process. Second to investigate how passivation and annealing affects the illumination sensitivity and finally attempt to explain the observed effects. Non-passivated materials as well as samples passivated by two different silicon nitride $\left(\mathrm{SiN}_{\mathrm{x}}\right)$ passivation processes are investigated. The electrical characterization spans from pure material transport measurements to different HFET characterization measurements, making it possible to trace the effect on the device level back to measurements of fundamental heterostructure characteristics. Investigated illumination conditions in this paper are white light illumination, no illumination, and partial spectrum illumination through different optical filters. Using the results from Hall characterization, a theory providing a qualitative explanation to the different illumination sensitivities is presented.

The report is divided into eight sections. Section II describes fabrication and characterization of test structures and devices. Section III presents the effect of PPC on Hall measurements and basic heterostructure transport characteristics. In Sec. IV, the Hall measurements are analyzed using a two layer conduction model which is first motivated by capacitancevoltage $[C(V)]$ charge analysis. Section $V$ presents the illumination sensitivity of the HFETs. Section VI presents a theoretical model for qualitative explanation of the PPC and the cause of the difference in sensitivity for the different samples. In Sec. VII, the results are further discussed. Finally, Sec. VIII presents the conclusions of this investigation. 


\section{EXPERIMENT}

Three samples from the same epitaxial wafer were used to fabricate HFETs and metal-insulator-semiconducting-heterostructure (MISH) structures. A schematic of the devices and epitaxial layers is shown in Fig. 1. The epi-layers were grown on a 3-in. semi-insulating $4 \mathrm{H}$ silicon carbide substrate. From bottom to top the layers are AlN nucleation layer, $2 \mu \mathrm{m}$ GaN buffer/ channel, $22 \mathrm{~nm} \mathrm{Al}_{0.14} \mathrm{Ga}_{0.86} \mathrm{~N}$ barrier, and $3 \mathrm{~nm} \mathrm{GaN}$ cap.

All HFET characteristics presented in this report were measured on two-finger devices, with a total gate periphery of $100 \mu \mathrm{m}$. The gate was $2 \mu \mathrm{m}$ long and placed in the center of a $6 \mu \mathrm{m}$ source drain separation. The MISH capacitors are circular pads with radii $100 \mu \mathrm{m}$ placed inside a circular ohmic contact opening. For the drift mobility extraction in Sec. IV, a FET with the passivation layer left un-etched below the gate (MISHFET) was characterized. The device has a gate length $10 \mu \mathrm{m}$ and drain source separation of $20 \mu \mathrm{m}$.

\section{A. Fabrication}

Four different samples are investigated in this report. The first sample, designated $R$, is a reference sample with a large van der Pauw (VDP) structure and non-annealed Gallium ohmic contacts. ${ }^{12}$ Three other HFET samples were processed, one without passivation, one passivated with reactively sputtered silicon nitride, and one passivated with LPCVD silicon nitride. These three samples are designated $N P, R S$, and $L P$, respectively. The HFET fabrication process utilizing the reactively sputtered silicon nitride process has been described previously. ${ }^{13,14}$ Shortly summarized the fabrication of the $R S$ sample is definition of isolated mesa structures by dry etching, deposition, and annealing of $\mathrm{Ti} / \mathrm{Al} / \mathrm{Ni} / \mathrm{Au}$ for ohmic contacts, passivation, gate passivation opening by $\mathrm{NF}_{3}$-based dry etching, Ni/Au gate deposition. For the processing of the $L P$ sample, there is one significant difference; the LPCVD $\operatorname{SiN}_{\mathrm{x}}$ is deposited before the ohmic contact process (to prevent metal contamination of the LPCVD furnace). This means that the heterostructure is protected by the passivation during ohmic contact process. The ohmic contact rapid thermal annealing (RTA) step is a two step annealing process, $30 \mathrm{~s}$ at $700^{\circ} \mathrm{C}$ and $30 \mathrm{~s}$ at $800^{\circ} \mathrm{C}$, in a nitrogen ambient. The LPCVD

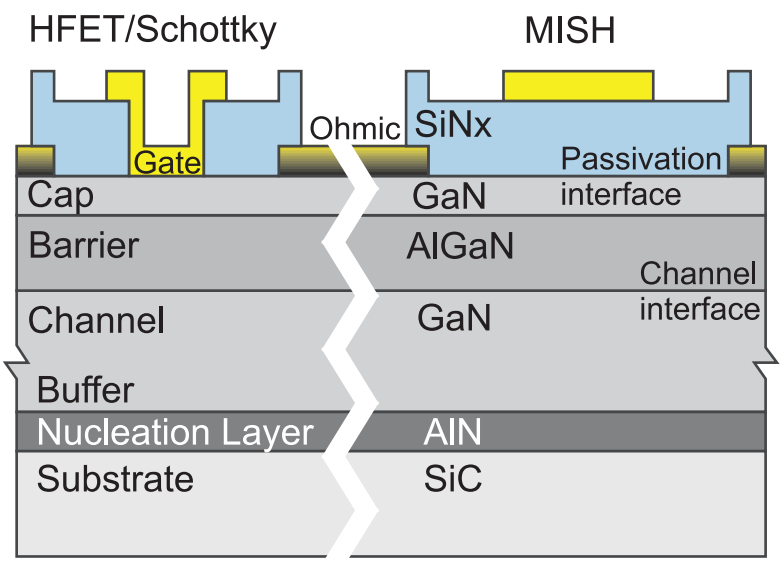

FIG. 1. Schematic cross-section of epitaxial layer describing the general layout of HFETs and MISH structures. The passivation interface and channel interface are defined as the passivation/cap and barrier/channel interfaces, respectively. tube-furnace is manufactured by Centrotherm and the deposition parameters are $\mathrm{SiH}_{2} \mathrm{Cl}_{2}$ and $\mathrm{NH}_{3}$ precursors at flow ratio $4: 1$, with deposition temperature and pressure of $770^{\circ} \mathrm{C}$ and 250 mTorr, respectively. The RS $\mathrm{SiN}_{\mathrm{x}}$ was $80 \mathrm{~nm}$ thick and had a refractive index of 1.99. The LPCVD $\operatorname{SiN}_{x}$ was $42 \mathrm{~nm}$ thick and had a refractive index of 2.15. The passivation layers were also deposited on fused silica and the transmittance of the films was measured using a Wollam M2000 ellipsometer; the results are reported in Sec. III.

\section{B. Characterization}

A Bio-Rad/Nanometrics HL5500 Hall characterization setup was used to measure sheet resistance $\left(R_{\mathrm{sh}}\right)$, electron sheet density $\left(n_{\mathrm{s}}\right)$, and electron Hall mobility $\left(\mu_{\mathrm{H}}\right)$. The Hall measurements started with the sample being continuously illuminated by fluorescent light. Measurements were then performed as a function of duration since suspending the illumination. DC characterization was performed using an HP 4145B Semiconductor Parameter Analyzer. C(V) characteristics of MISH capacitors and MISHFETs were measured using HP4284 LCR meter. Pulsed I(V) measurements were performed using an Accent technologies DiVA 225 with pulse length of $500 \mathrm{~ns}$ and a pulse separation of $2 \mathrm{~ms}$. Small signal S-parameters were measured using an Agilent E8361 Vector Network Analyzer and load-pull measurements were performed using the setup described by Thorsell and Andersson ${ }^{15}$ The illumination sensitivity, as defined by $\left|X_{\text {illuminated }}-X_{\text {dark }}\right| / X_{\text {illuminated }}$, can then be extracted for any measured quantity $(X)$.

Illumination sources of both incandescent and fluorescent types have been used. More specifically the incandescent light source was a $60 \mathrm{~W}$ light bulb with color temperature $2800 \mathrm{~K}$ which was placed $0.3 \mathrm{~m}$ from the sample. The fluorescent source was a tri-color $58 \mathrm{~W}$ fluorescent tube light with color temperature $3000 \mathrm{~K}$ placed at a distance of $1.5 \mathrm{~m}$ from the sample. Figure 2 plots the spectral irradiance of the two lamp setups, measured using a StellarNet Inc. Blue-wave spectrometer. The incandescent lamp provides a continuous black-body type radiation which has relatively high intensity in the red and infrared region of the spectrum. The spectral power distribution of the fluorescent lamp is made up of several peaks, where the peaks at $315 \mathrm{~nm}$ and $365 \mathrm{~nm}$ are of special interest to the investigation in this paper.

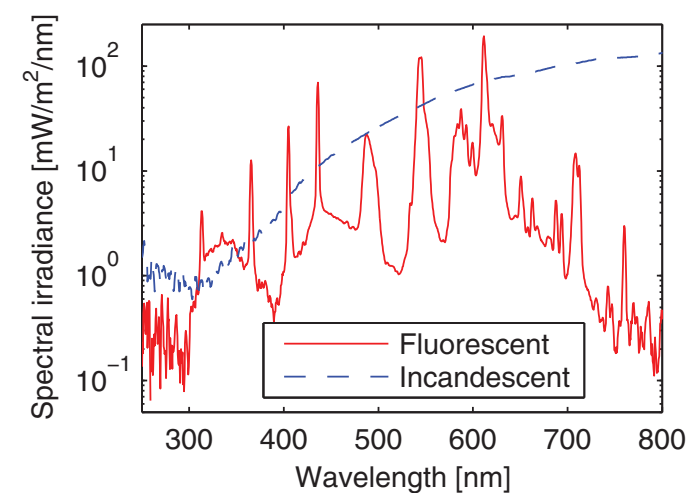

FIG. 2. Comparison of the spectral irradiance of the fluorescent and incandescent lamp setups. 
The optical filters were colored-glass long-pass filters manufactured by SCHOTT, which have a transmittance of 0.8-0.9 for wavelengths $(\lambda)$ longer than the filter cut-off wavelength $\left(\lambda_{\mathrm{C}}\right)$ and about 0 transmittance for $\lambda<\lambda_{\mathrm{C}}$. The wavelength range for transition from 0 to 0.8 transmittance is approximately $\lambda_{\mathrm{C}} \pm 15 \mathrm{~nm}$.

\section{RESULTS: HALL AND PPC}

\section{A. Hall}

Figure 3 presents measured Hall parameters $\left(R_{\mathrm{sh}}, n_{\text {meas }}\right.$, and $\mu_{\text {meas }}$ ) obtained under different illumination conditions. In this and all remaining figures, the open symbols represent measurements under continuous illumination, the partially filled and filled symbols are measured $1 \mathrm{~min}$, respectively, longer time after suspending illumination. The inset shows the evolution of $n_{\text {meas }}$ as the illumination is suspended at $\mathrm{t}=0$. The general behavior is the same for all samples; initially $R_{\mathrm{sh}}$ increases rapidly, the rate of increase declines, and eventually a stable $R_{\mathrm{sh}}$ is reached. The reason for the reduction of $R_{\mathrm{sh}}$ is predominately caused by a decrease of $n_{\text {meas }}$, as the graph in the inset shows. For $N P$ and $R S$, there is also a reduction of the mobility while the mobility is almost the same for $R$ and $L P$ (Table IV in Sec. V). The $R, N P, R S$, and $L P$ samples were kept in dark for a time period of up to 9, 88,15 , and $55 \mathrm{~h}$, respectively. After this the samples were reilluminated, causing $R_{\mathrm{sh}}$ and $n_{\text {meas }}$ to recover fully to the previously illuminated values and the same illuminated-to-dark behavior could then be measured repeatedly. However, the time constant of the illuminated to dark transition is much longer compared to that of the reverse process, hours compared to tens of seconds. The illumination sensitivity and duration until a stable measurement can be extracted varies greatly between the samples. The sensitivity in $R_{\mathrm{sh}}$ is $25 \%$, $135 \%, 16 \%$, and $3 \%$ for $R, N P, R S$, and $L P$, respectively, and the time until stable Hall measurement can be extracted is $8,82,4$, and $3 \mathrm{~h}$, respectively.

Comparing $N P$ and $R$ shows that the ohmic contact fabrication process with the annealing is responsible for a very

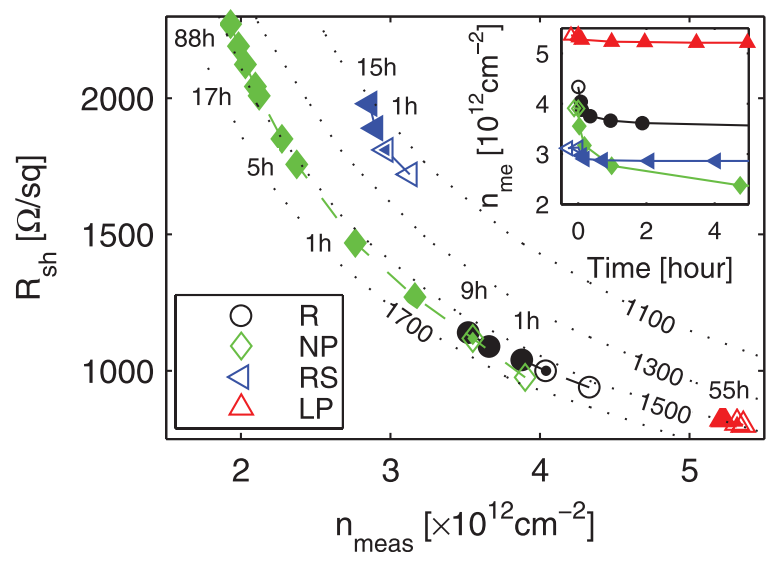

FIG. 3. Hall characteristics, the open symbols are measured with illumination and the filled symbols represent measurement after different durations in dark. The duration in dark at some points is given by the numbers next to the data point. The $\mu_{\text {meas }}$ is represented by the contour lines. $n_{\text {meas }}$ versus time in dark is presented in the inset.



FIG. 4. $G_{\text {PPC }}$ extracted from the presented lumped model versus duration in dark. The inset shows a magnified view of the recovery from dark to illuminated when the illumination is activated at $0 \mathrm{~s}$.

large increase of $R_{\mathrm{sh}}$. The magnitude of the effect is only fully comprehended when comparing the non-illuminated values. Comparing illuminated values of $N P$ and $R S$ results in only $3 \%$ higher $R_{\mathrm{sh}}$ of $N P$, while it is more than $100 \%$ higher when comparing dark values. Deposition of the RS $\mathrm{SiN}_{\mathrm{x}}$ increases $R_{\mathrm{sh}}$, at least when comparing with illuminated values of $N P$. When comparing dark values, the $R_{\mathrm{sh}}$ compared to $N P$ is improved by the reactively sputtered passivation. It is, however, obvious that deposition of the RS passivation has reduced the mobility in the structure. $L P$ has not been affected in the same way by the annealing since depositing the passivation before the RTA prevents the large $R_{\mathrm{sh}}$ increase due to the annealing. ${ }^{16}$ The $L P$ sample has high $n_{\mathrm{s}}$ and high $\mu_{\mathrm{H}}$ and is only minutely affected by differences in illumination.

\section{B. Persistent photoconductivity}

The conductance due to the PPC $\left(G_{\mathrm{PPC}}\right)$ is plotted in Fig. 4, where $G_{\mathrm{PPC}}$ in parallel with the dark level conductance $\left(G_{0}\right)$ provides the total material conductance (a schematic is drawn inside the figure). The linear dependence of $G_{\mathrm{PPC}}$ versus logarithmic time scale can be fitted using a stretched exponential function ${ }^{6} G_{\mathrm{PPC}}=G_{\text {ill }} e^{-(t / \tau)^{\beta}}$, where $G_{\text {ill }}$ is the $G_{\text {PPC }}$ for the continuously illuminated material. By fitting the curve to the measured data, the equation parameters that are presented in Table I have been extracted. The sharp steps for each curve represents the recovery of $G_{\mathrm{PPC}}$ back to $G_{\text {ill }}$ when the lamp is turned on again. The inset of Fig. 4 plots a magnified view of the recovery where the timeaxis has been shifted so that $0 \mathrm{~s}$ represents lamp ignition. The majority of $G_{\mathrm{PPC}}$ is recovered within the first five seconds.

TABLE I. Extracted $G_{\mathrm{PPC}}$ decay parameters.

\begin{tabular}{lccr}
\hline \hline & $G_{\text {ill }}(\mu \mathrm{S} \cdot \mathrm{sq})$ & $\beta$ & $\tau(\mathrm{s})$ \\
\hline$R$ & 350 & 0.35 & 500 \\
$N P$ & 560 & 0.26 & 4000 \\
$R S$ & 90 & 0.35 & 200 \\
$L P$ & 30 & 0.35 & 100 \\
\hline \hline
\end{tabular}


$G_{\mathrm{PPC}}$ versus $\lambda_{\mathrm{C}}$ for the $N P$ sample is shown in Fig. 5. A sample left in darkness for several hours was illuminated with a incandescent light source through the different filters. The sample was illuminated with the filtered light for $5 \mathrm{~min}$ before each $R_{\mathrm{sh}}$ measurement. The light was then switched off and the procedure was repeated with a filter with a shorter $\lambda_{\mathrm{C}} . G_{\mathrm{PPC}}$ is seen to increase sharply with $\lambda_{\mathrm{C}}$ between 395 and $335 \mathrm{~nm}$ (3.1 and $3.7 \mathrm{eV}$, respectively), which spans the energy bandgaps of $\mathrm{GaN}(3.4 \mathrm{eV}, 365 \mathrm{~nm})$ and $\mathrm{Al}_{0.14} \mathrm{Ga}_{0.86} \mathrm{~N}(3.64 \mathrm{eV}, 340 \mathrm{~nm}) .{ }^{17}$ There is some uncertainty in the bandgap of $\mathrm{AlGaN}$ due to a large spread in reported bandgap bowing parameters. ${ }^{29}$ The only difference experienced when using an incandescent light source is that $G_{\text {ill }}$ is slightly lower and that the transition from dark to illuminated is slower (the decay time constant for the transition from illumination to dark is approximately the same). The difference is explained by that the fluorescent lamp has a higher intensity in the 310 to $365 \mathrm{~nm}$ wavelength range, as can be seen in Fig. 2.

The transmittance of the passivations are plotted on the right axis of Fig. 5. The fused silica $\left(\mathrm{SiO}_{2}\right)$ wafer, used as carrier substrate for the $\mathrm{SiN}_{\mathrm{x}}$ depositions, has a transmittance of around 0.9 for the entire wavelength range. A sample deposited with the RS $\mathrm{SiN}_{\mathrm{x}}$ has a transmittance of about 0.8 . The sample deposited with $\mathrm{LPCVD} \mathrm{SiN}_{\mathrm{x}}$ is seen to have a generally lower transmittance and it is less transparent in the short wavelength region where the PPC effect is the largest. Thus, at least $40 \%$ of the reduced illumination sensitivity of $L P$ can be directly correlated to a lower transmission in the blue to ultra-violet range of the spectrum.

\section{TWO LAYER CONDUCTION}

In this section, the Hall data are analyzed using a model where conduction occurs in two layers. The reason for considering two layer conduction is first motivated by analyzing the charge distribution in the passivated materials.

\section{A. MISH capacitance-voltage characteristics}

The location and amount of charge in the structures can be extracted from $\mathrm{C}(\mathrm{V})$ measurements of MISH capacitor

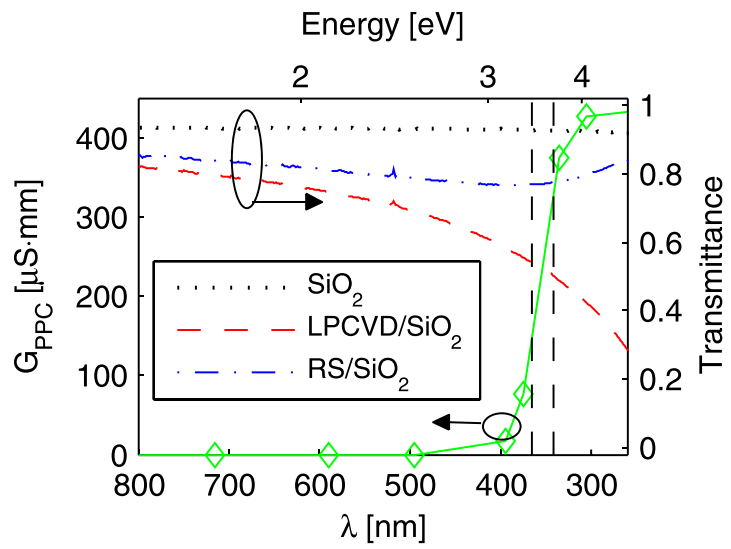

FIG. 5. $G_{\mathrm{PPC}}$ for $N P$ versus photon wavelength and energy. The transmittance of the films are plotted on the right axis. The bandgaps of $\mathrm{Al}_{0.14} \mathrm{Ga}_{0.86} \mathrm{~N}$ and $\mathrm{GaN}$ are represented by the vertical lines. structures. The capacitances of $R S$ and $L P$ extracted at a measurement signal frequency of $1 \mathrm{kHz}$ are shown in Fig. 6. The voltage is swept from depletion $(-14 \mathrm{~V})$ to passivation accumulation at $10 \mathrm{~V}$ and back to depletion. The generally lower capacitance of $R S$ is due to the thicker passivation. For $R S$ a measurement with backside illumination by a fluorescent light source is also plotted; for $L P$ the backside illuminated characteristics are omitted since they are almost identical to the non-illuminated characteristics. The $100 \mathrm{kHz}$ characteristics of the backside illuminated sample is used since measurement with lower test signal resulted in noisy data. These characteristics can be used to extract much information about states at the passivation interface. ${ }^{18}$ However, obtaining a detailed picture of the passivation interface is outside the scope of this investigation. It will only be shortly stated that the hysteresis between sweep directions $(\Delta \mathrm{V})$ indicates that there are traps at the passivation interface of both samples (a much higher density of states for $R S$ ).

For this investigation, it is interesting to extract the charge density and distribution of charge, which can be done by integrating the $\mathrm{C}(\mathrm{V})$ characteristic. An equivalent number density of charge $\left(n_{\mathrm{CV}}\right)$ is obtained using $n_{\mathrm{CV}}=1 / q \int C d V$, where $q$ is the elementary charge. Another parameter of interest is the voltage for barrier accumulation $\left(V_{\mathrm{BA}}\right)$, at which there starts to be a significant accumulation of electrons in the barrier region. An integration from depletion to $V_{\mathrm{BA}}$, designated $n_{\mathrm{CV}, V_{\mathrm{BA}}}$, is a measure of how many electrons can be filled in the channel before there is significant spill-over of electrons to the barrier. It is also likely that if a non-gated heterostructure has a higher sheet density than $n_{\mathrm{CV}, V_{\mathrm{BA}}}$, there will be electrons not only in the channel. The formation of a surface quantum well for GaN/AlGaN/GaN heterostructures has been reported by several groups. ${ }^{19,20}$ In this report, it is assumed that when the electrons are no longer accumulating in the channel they will most likely accumulate in a cap layer potential well. Hence, in the remainder of the paper, the mobile electrons are assumed to be either in the channel or in the cap.

An integration from depletion to $0 \mathrm{~V}$, designated $n_{\mathrm{CV}, 0}$, provides a measure of $n_{\mathrm{s}}$ in the non-gated heterostructure. The validity of this comparison depends on how much the

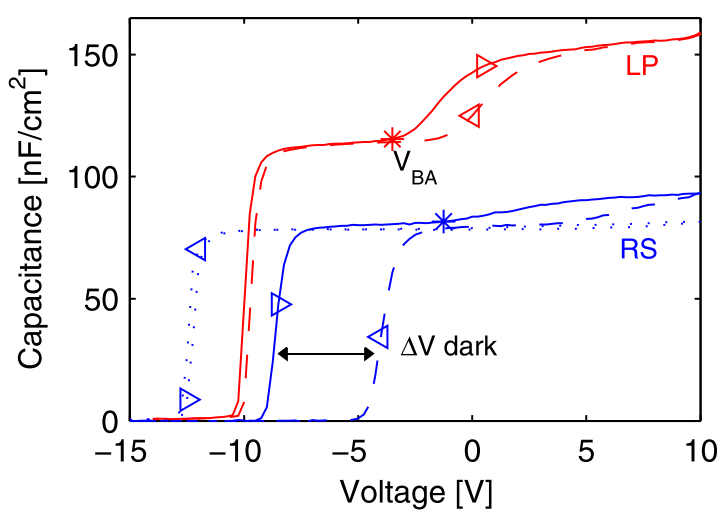

FIG. 6. Capacitance of MISH structures measured with a test signal frequency of $1 \mathrm{kHz}$. The solid and the dashed lines are the forward and reverse sweeps, respectively, measured without illumination. The dotted lines are the $100 \mathrm{kHz}$ forward and reverse sweeps for $R S$ with backside illumination. 
TABLE II. Barrier accumulation voltage and charge densities extracted by $\mathrm{C}(\mathrm{V})$ measurements. The rightmost column presents the calculated channel confinement ratios.

\begin{tabular}{lcccc}
\hline \hline & $V_{\mathrm{BA}}(\mathrm{V})$ & $n_{\mathrm{CV}, V_{\mathrm{BA}}}\left(\mathrm{cm}^{-2}\right)$ & $n_{\mathrm{CV}, 0}\left(\mathrm{~cm}^{-2}\right)$ & $C C R$ \\
\hline$R S$ & -1.2 & $3.8 \times 10^{12}$ & $4.3-6.5 \times 10^{12}$ & $0.58-0.88$ \\
$L P$ & -3.5 & $4.7 \times 10^{12}$ & $6.9 \times 10^{12}$ & 0.68 \\
\hline \hline
\end{tabular}

metal changes the surface potential of the passivation. According to the calculations reported by Cook et al., ${ }^{21}$ the Fermi level of $\mathrm{Si}_{3} \mathrm{~N}_{4}$ deposited on $\mathrm{GaN}$ is $5.6 \mathrm{eV}$ below the vacuum level, which is close to the $5.1 \mathrm{eV}$ work function of Nickel. $^{22}$ Hence, the band alignment of the two cases is roughly comparable and the charge extracted by integrating the $\mathrm{C}(\mathrm{V})$ is comparable to the charge in the non-metalized material. The charge extracted from the $\mathrm{C}(\mathrm{V})$ is reported in Table II, where the concept of channel confinement ratio $(C C R)$ is also introduced. $C C R$ is the ratio of electrons in the channel to the total electron density, i.e., $n_{\mathrm{CV}, \mathrm{VBA}} / n_{\mathrm{CV}, 0}$.

For the non-illuminated characteristics of $R S, n_{\mathrm{CV}, V_{\mathrm{BA}}}$ is lower than the equivalent value for $L P$ which indicates that in $R S$ the channel cannot be filled with as many electrons before the electrons start to accumulate in the cap. The hysteresis, $\Delta \mathrm{V}$, of the dark measurement is equivalent to about $2 \times 10^{12} \mathrm{~cm}^{-2}$. Furthermore, the voltage shift between the backside illuminated and non-illuminated characteristics is equivalent to $1.8 \times 10^{12} \mathrm{~cm}^{-2}$. The hysteresis as well as the shift for different measurement conditions implicates a large quantity of trapped electrons that are only emitted with assistance of illumination. The large difference depending on measurement conditions for $R S$ is reflected by the range of vales reported in Table II.

\section{B. Two layer analysis}

The accumulation layer in the cap will form a parallel conduction path that will make the measured Hall parameters reported in Fig. 3 dubious since these were extracted assuming a uniform material. Equations (1) and (2), as reported by Petritz ${ }^{23}$, can be used to relate the experimentally obtained $\mu_{\text {meas }}$ and $n_{\text {meas }}$ to individual layer mobilities and sheet densities of a two layer conduction material

$$
\begin{gathered}
\mu_{\text {meas }}=\frac{n_{\text {cap }} \mu_{\text {cap }}^{2}+n_{\text {chn }} \mu_{\text {chn }}^{2}}{n_{\text {cap }} \mu_{\text {cap }}+n_{\text {chn }} \mu_{\text {chn }}}, \\
n_{\text {meas }}=\frac{\left(n_{\text {cap }} \mu_{\text {cap }}+n_{\text {chn }} \mu_{\text {chn }}\right)^{2}}{n_{\text {cap }} \mu_{\text {cap }}^{2}+n_{\text {chn }} \mu_{\text {chn }}^{2}},
\end{gathered}
$$

where $n_{\text {cap }}, \mu_{\text {cap }}$ and $n_{\text {chn }}, \mu_{\text {chn }}$ are the sheet densities and Hall mobilities in the cap and channel, respectively.

Also, the mobility in each layer has to be adjusted due to the influence of Coulomb and interface scattering. For low $n$, the mobility is expected to be lower due to a large contribution of Coulomb scattering. ${ }^{24}$ For high $n_{\mathrm{s}}$, the mobility is expected to be lower due to increased influence of interface scattering. ${ }^{25}$ To investigate the influence of Coulomb and interface scattering the drift mobility versus $n_{\mathrm{s}}$ for a

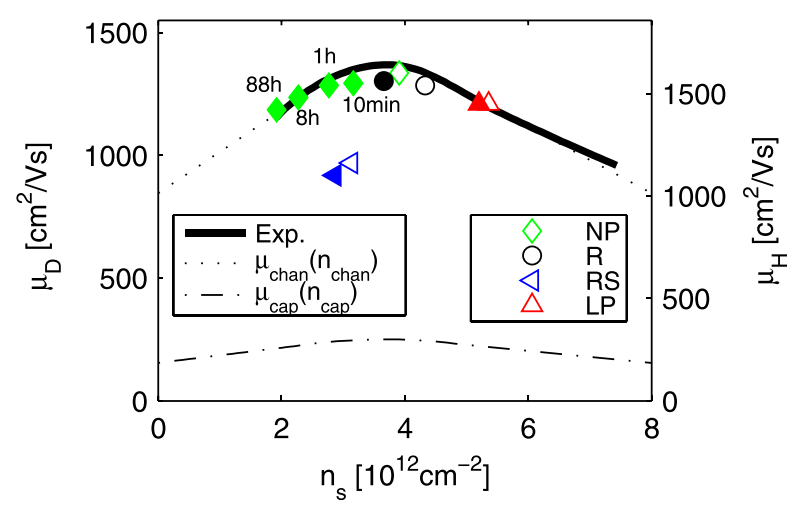

FIG. 7. $\mu_{\mathrm{D}}$ (left axis) and $\mu_{\mathrm{H}}=1.19 \times \mu_{\mathrm{D}}$ (right axis) versus carrier density. The measured illuminated (open symbols) and dark (filled symbols) Hall mobilities are plotted versus $n_{\text {meas }}$. For $N P$, the illuminated measurement and measurements $10 \mathrm{~min}$ to approximately 3 days after suspending illumination are plotted.

MISHFET on $L P$ was extracted by correlating the resistance versus gate bias to the sheet density as obtained by $\mathrm{C}(\mathrm{V})$ integration. $^{24,25}$ The solid line in Fig. 7 represents the extracted drift mobility versus electron density.

A model for the channel mobility versus channel sheet density $\left[\mu_{\mathrm{chn}}\left(n_{c h n}\right)\right]$ is constructed as follows. The model is split into three intervals, one where it interpolates the measured data around the mobility peak and one interval each for high and low $n_{\mathrm{chn}}$, where the model linearly extrapolates the measured data. The relation between Hall and drift mobility is found by matching the different mobilities at $n_{\mathrm{chn}}=1.9$ $\times 10^{12} \mathrm{~cm}^{-2}$, which is equivalent to assuming that conduction is localized only to the channel for NP when it has been left in dark for a long time. Using this point to fit the curves results in a peak channel Hall mobility of $1630 \mathrm{~cm}^{2} / \mathrm{Vs}$. Since the peak channel drift mobility is $1370 \mathrm{~cm}^{2} / \mathrm{Vs}$, the resulting Hall factor is 1.19 , which is in agreement with 1.2 reported by Shur et al. ${ }^{26}$

Unfortunately, there is no similar straight-forward method to extract $\mu_{\text {cap }}\left(n_{\text {cap }}\right)$, since there is no way to isolate conduction only in the cap layer. In lack of a better model, the $\mu_{\mathrm{chn}}\left(n_{c h n}\right)$ relation was simply rescaled with a peak mobility of $300 \mathrm{~cm}^{2} / \mathrm{Vs}$ (this value is used as a fitting variable). The cap mobility model is inherently flawed since the cap is only $3 \mathrm{~nm}$ thick. Hence, an electron will always be in close proximity to two interfaces with large polarization charge densities, having an effect on both Coulomb and interface scattering. However, we claim that the model is appropriately accurate to support the qualitative discussion in this report.

The measured Hall parameters have been plotted against the Hall mobility on the right axis of Fig. 7. The three leftmost NP points are consistent with conduction localized only in the channel. The remaining values can be modelled by assuming a combination of channel and cap conduction.

The two plots of Fig. 8 are relating the measured Hall parameters to the constructed model. In the left plot, $\mu_{\text {meas }}$ is plotted versus different $C C R=n_{\mathrm{chn}} / n_{\text {tot }}$ where the lines represent four different $n_{\text {tot }}=n_{\text {chn }}+n_{\text {cap }}$. In the right plot, $n_{\text {meas }}$ is plotted versus $C C R$ for different $n_{\text {tot }}$. The data points representing the non-illuminated experimental Hall data have 



FIG. 8. $\mu_{\text {meas }}$ (left) and $n_{\text {meas }}$ (right) for different $n_{\mathrm{s}}$ and channel confinement ratios. The lines represent four different $n_{\text {tot }}$ and the Hall data extracted in darkness has been inserted in the plot.

been inserted at a $C C R$ and $n_{\text {tot }}$ that simultaneously provides the best fit to the measured Hall characteristics. The extracted $C C R$ and $n_{\text {tot }}$, also for illuminated measurements, are presented in Table III. Considering the assumptions regarding conduction in the cap the error margin of $C C R$ is at least 0.1 , it is even larger for low $C C R$ since the extraction is to a greater extent affected by the assumptions made for the cap mobility.

For $L P$, the value is supported by a $C C R$ of 0.7 extracted from MISH capacitor measurements. For $R S$, the $C C R$ is much lower than the 0.6-0.9 indicated by the MISH C(V) measurement. It is not obvious which value is more trustworthy since the $\mathrm{C}(\mathrm{V})$ extraction is perturbed by different interface states while the two-layer extraction is heavily influenced by the flawed cap mobility model. Even though the results differ, both extraction methods indicate significant quantities of electrons in the cap layer which is of importance for the following discussion.

Assuming that the presented model is correct, the number of electrons generated by illumination can be extracted from Table III. The illumination is resulting in a increase of $2.1 \times 10^{12} \mathrm{~cm}^{-2}$ for $N P, 0.3 \times 10^{12} \mathrm{~cm}^{-2}$ for $R$ and $R S$, and $0.1 \times 10^{12} \mathrm{~cm}^{-2}$ for $L P$. The lower increase of $L P$ compared to $R$ and $R S$ can be explained by the lower transmittance of the LPCVD silicon nitride. The increase for $L P$ becomes almost insignificant compared to the large dark level of $n_{\text {tot }}$.

To summarize, the most significant difference between the three materials is that NP differs from the two other samples in the respect that it has negligible concentration of electrons in the cap after about 30 min in darkness.

Section V puts focus on the practical effect of the illumination on HFET devices. As a general statement, an increasing resistance of devices is expected when the illumination is suspended due to the PPC.

TABLE III. Extracted two layer analysis parameters extracted for illuminated and (dark) measurements.

\begin{tabular}{lcc}
\hline \hline & $C C R$ & $n_{\mathrm{tot}}\left(\times 10^{12} \mathrm{~cm}^{-2}\right)$ \\
\hline$R$ & $0.89(\mathbf{0 . 7 9})$ & $4.7(\mathbf{4 . 4})$ \\
$N P$ & $0.95(\mathbf{1 . 0 0})$ & $4.0(\mathbf{1 . 9})$ \\
$R S$ & $0.41(\mathbf{0 . 3 7})$ & $4.8(\mathbf{4 . 5})$ \\
$L P$ & $0.86(\mathbf{0 . 8 3})$ & $6.0(\mathbf{5 . 9})$ \\
\hline \hline
\end{tabular}

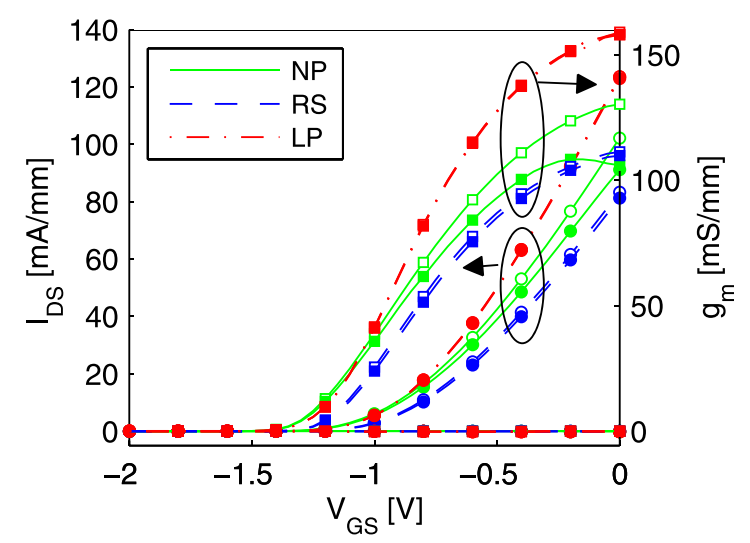

FIG. 9. Drain current (left) and transconductance (right) versus gate voltage for $V_{\mathrm{DS}}=0$ and $10 \mathrm{~V}$. Open symbols are continuously illuminated and the closed symbols are measured in dark.

\section{RESULTS: HFETS}

During measurements of the HFETs, the illumination was from a ceiling panel fluorescent lamp and the dark measurement were extracted, at least, $30 \mathrm{~min}$ after suspending illumination. This duration in dark provides apparently stable results for $R S$ and $L P$ but represents about $70 \%$ of the $G_{\mathrm{PPC}}$ decay for $N P$.

\section{A. DC and small signal characteristics}

Figure 9 presents the transfer characteristics of the HFET samples. The transconductance $\left(g_{\mathrm{m}}\right)$ and saturated current at $V_{\mathrm{GS}}=0 \mathrm{~V}\left(I_{\mathrm{DSS}}\right)$ are presented in Table IV. The pinch-off voltage is illumination invariant due to the shadowing effect of the gate and the gate current is illumination invariant as well. The pinch-off voltage is almost identical for all samples, indicating that deposition of the Schottky metal results in the same surface pinning, independent of the processing prior to the gate deposition step. This is also an indication that the heterostructure is not severely structurally damaged during any of the process steps investigated in this report. The illumination sensitivity is $20 \%, 2 \%$, and $0 \%$ for $N P, R S$, and $L P$ respectively. The sensitivity of $N P$ would probably end up at around $30 \%$ if it was left in darkness for a much longer time.

TABLE IV. Collection of illuminated and (dark) characteristics for different samples. The Hall parameters of $\mathrm{R}: R_{\mathrm{sh}}=939(\mathbf{1 1 4 0}) \Omega / \mathrm{sq} ; n_{\text {meas }}$ $=4.33(\mathbf{3 . 5 2}) \mathrm{cm}^{-2} ; \mu_{\text {meas }}=1540(\mathbf{1 5 6 0}) \mathrm{cm}^{2} / \mathrm{Vs}$.

\begin{tabular}{lccc}
\hline \hline & $N P$ & $R S$ & $L P$ \\
\hline$R_{\text {sh }}(\Omega / \mathrm{sq})$ & $976(\mathbf{2 2 9 3})$ & $1720(\mathbf{1 9 9 0})$ & $801(\mathbf{8 2 5})$ \\
$n_{\text {meas }}\left(\times 10^{12} \mathrm{~cm}^{-2}\right)$ & $3.91(\mathbf{1 . 9 2})$ & $3.13(\mathbf{2 . 8 6})$ & $5.36(\mathbf{5 . 2 0})$ \\
$\mu_{\text {meas }}\left(\mathrm{cm}^{2} / \mathrm{Vs}\right)$ & $1620(\mathbf{1 4 2 0})$ & $1160(\mathbf{1 1 0 0})$ & $1450(\mathbf{1 4 5 0})$ \\
$g_{\mathrm{m}}(\mathrm{mS} / \mathrm{mm})$ & $130(\mathbf{1 0 8})$ & $110(\mathbf{1 1 0})$ & $158(\mathbf{1 5 8})$ \\
$\mathrm{I}_{\mathrm{DSS}}(\mathrm{mA} / \mathrm{mm})$ & $102(\mathbf{9 4})$ & $83(\mathbf{8 1})$ & $124(\mathbf{1 2 4})$ \\
$f_{\mathrm{T}}(\mathrm{GHz})$ & $3.7(\mathbf{2 . 2})$ & $4.8(\mathbf{4 . 7})$ & $6.0(\mathbf{6 . 0})$ \\
$f_{\text {max }}(\mathrm{GHz})$ & $14.8(\mathbf{1 0 . 4})$ & $23.6(\mathbf{2 1 . 6})$ & $43.5(\mathbf{4 3 . 4})$ \\
$P_{\text {out }}(\mathrm{dBm})$ & $10.4(\mathbf{9 . 7})$ & $14.7(\mathbf{1 4 . 2})$ & $20.2(\mathbf{2 0 . 2})$ \\
$\mathrm{Gain}(\mathrm{dB})$ & $1.1(\mathbf{0 . 2})$ & $2.1(\mathbf{1 . 8})$ & $7.6(\mathbf{7 . 6})$ \\
$\eta(\%)$ & $10(\mathbf{9})$ & $24(\mathbf{2 3})$ & $37(\mathbf{3 6})$ \\
\hline \hline
\end{tabular}


Cut-off frequency $\left(f_{\mathrm{T}}\right)$ and maximum frequency of oscillation $\left(f_{\max }\right)$ were extracted for HFETs under both illuminated and dark conditions. The values are presented in Table IV. The PPC will have an effect on the access resistances, explaining the large effect of illumination on $f_{\mathrm{T}}$ and $f_{\max }$ of $N P$. For $R S$, the effect is much smaller. The $f_{\mathrm{T}} / f_{\max }$ of $6 / 43 \mathrm{GHz}$ of $L P$ are comparable with previously published fat-gate devices. ${ }^{27}$

\section{B. Pulsed I(V) and load-pull characteristics}

Figure 10 presents the pulsed $\mathrm{I}(\mathrm{V})$ characteristic for the HFET samples at $V_{\mathrm{GS}}=0 \mathrm{~V}$. The curves are measured using two different quiescent biases, $Q B\left(V_{\mathrm{GS}}, V_{\mathrm{DS}}\right)$. A reference bias $Q B_{0}=Q B(0,0)$ and a class-B like bias $Q B_{\mathrm{CB}}=Q B(-5$, $25)$ are plotted in the figure. The current is almost totally collapsed for $N P$. When illuminated, the relative current collapse for $R S$ and $L P$ are almost identical. However, when not illuminated there is a significant additional collapse for $R S$ around the knee-voltage.

The $Q B_{\mathrm{CB}}$ characteristics of $R S$ measured when illuminated through the different optical filters are also plotted. The illuminated pulsed characteristic is reproduced with $\lambda_{\mathrm{C}} \leq 395 \mathrm{~nm}$ and the non-illuminated characteristic is reproduced with $\lambda_{\mathrm{C}} \geq 715 \mathrm{~nm}$. Hence, the photon energies responsible for reducing current collapse are different from those affecting the electron sheet density. Wavelengths between 395 and $715 \mathrm{~nm}$ correspond to energies between 3.1 and $1.7 \mathrm{eV}$, approximately the lower half of the bandgap of GaN. The change in current collapse with different illumination is instantaneous, i.e., no successive change with duration for either an illuminated or non-illuminated device. The fact that the illumination can impede current collapse is well documented. ${ }^{9}$ The improvement of the dispersion under illumination is due to photon-assisted emission of electrons trapped close to the gate edge.

Load pull measurements were performed on devices under both illuminated and dark conditions. The devices were biased and matched for maximum output power. The saturated output power $\left(P_{\text {out }}\right)$ and drain efficiency $(\eta)$ are reported in Table IV. The power results are extremely poor

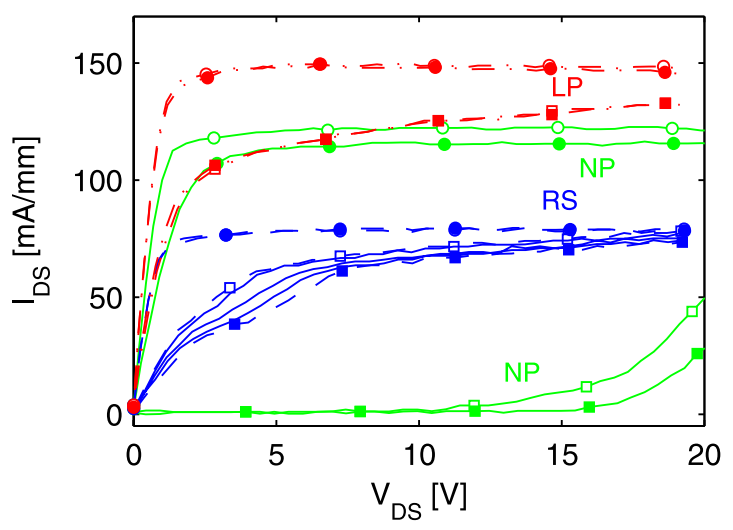

FIG. 10. Pulsed characteristics for $N P$ (green, solid), $R S$ (blue, dashed), and $L P$ (red,dash-dotted). The open symbols are illuminated and the closed nonilluminated conditions. Circles represent $Q B_{0}$ and squares $Q B_{\mathrm{CB}}$. The three additional $Q B_{\mathrm{CB}}$ curves for $R S$ are illuminated through filters with $\lambda_{\mathrm{C}}=590,530$, and $455 \mathrm{~nm}$, respectively (from lowest to highest current).
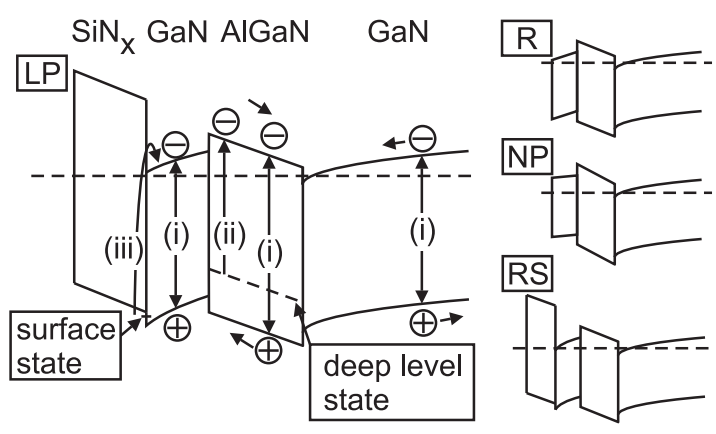

FIG. 11. Schematic band structures of the different samples; the thickness of the cap is greatly overexaggerated. The larger diagram represents the proposed scenario for $L P$ and also shows the forces experienced for photogenerated electrons and holes.

for $N P$ which was expected considering the large current collapse seen in the pulsed measurements. For $R S$, the device is significantly better but still quite poor performance. The $P_{\text {out }}=20 \mathrm{dBm}$ for $L P$ corresponds to a power density of $1 \mathrm{~W} / \mathrm{mm}$. Generally, the illumination effect is negligible for devices on $L P$, which is in correspondence with the Hall and $\mathrm{C}(\mathrm{V})$ results.

\section{THEORETICAL ANALYSIS}

In the following, we present a theory with reference to the schematic band diagrams of Fig. 11, the analysis is similar to that reported by Li et al. ${ }^{7}$ but with a different heterostructure. The extraction of the energy dependence of the PPC showed that it is illumination with an energy between 3.1 and $3.7 \mathrm{eV}$ that generates the PPC. The result is a bit different compared to several other reports, where the PPC is observed to be generated when radiated with significantly lower photon energy. The effect is then reported to be due to excitation of deep level states in the AlGaN. ${ }^{3,6,7}$ Since the energy interval for the PPC spans the bandgaps of both GaN and $\mathrm{Al}_{0.14} \mathrm{Ga}_{0.86} \mathrm{~N}$, we propose three different excitation/generation processes: (i) electron-hole pairs generated by photons with energies larger than the bandgap of $\mathrm{GaN}$ and possibly also AlGaN, (ii) electron excitation from deep level states in the AlGaN, and (iii) electron excitation from states at the surface or passivation interface.

In the case of electron-hole generation, the pairs will become spatially separated due to the electric fields caused by the polarization. The separation will be most prominent for generation in the channel/buffer and for pairs generated in the barrier, while electron-hole pairs that are generated in the cap will stay in the cap and have a very fast recombination due to being in close proximity to each other.

Electrons that are generated from deep level states in the AlGaN will fall into the channel. When illumination is suspended the recombination can be assumed to be quite slow but will probably be faster if there are electrons also in an accumulation layer in the cap since the deep level states in the barrier will be filled from the cap side, as well.

For electrons generated from a surface state, the time for recombination will depend on where the excited electron ends up. If the electron is transferred down to the channel the recombination can be very slow. Regardless of where the 
electron eventually ends up, it is obvious that having electrons in a cap accumulation layer will result in a much faster recombination when illumination is suspended.

\section{DISCUSSION}

The illumination sensitivity of HFETs are generally lower than the sensitivity of the materials. The main difference is that the gated heterostructure material is not sensitive to fromthe-top illumination. Furthermore, there is also an effect of proximity to ohmic contacts. For $R S$ and $L P$, the $G_{\mathrm{PPC}}$ was about $20 \%$ lower when extracted from contact pairs with ohmic contact separations of $5-30 \mu \mathrm{m}$ than for the $G_{\mathrm{PPC}}$ extracted from VDP structures. On the VDP structures, the ohmic contacts are at a distance of at least $150 \mu \mathrm{m}$ from the measured material. For $N P$, a large spread between measurement structures prevents a similar conclusion. The lower sensitivity seems reasonable considering that the ohmic metal covers large areas of material that are not affected by the illumination. Electrons generated in the proximity of the ohmic contact may diffuse into the ohmic contact area, thus reducing the effect of the generated electron. On the other hand, the time of the PPC decay was significantly longer (on the order of $50 \%$ ) for the material in proximity to the ohmic contact. The longer time is also an indication that the generated carriers have been spatially further separated from the generation point. Using $R S$ as an example, the difference in illumination sensitivity for different structures are $30 \%$ for VDP extracted $R_{\mathrm{sh}}, 10 \%$ in resistance between two ohmic contacts, and $1 \%$ to $5 \%$ in extracted HFET device characteristics.

Measurement on contact pairs with different contact spacing allowed for transfer length method extraction of the contact resistivity $\left(r_{\mathrm{c}}\right)$. For $R S$ and $L P$, the contact resistivities are practically illumination invariant at around $0.5 \Omega \mathrm{mm}$. For $N P$, the extractions are perturbed by a significant difference for the PPC decay of different contact pairs, even when the contact pairs are located right next to each other. The illumination sensitivity for $N P$ is always large but the magnitude of the $G_{\mathrm{PPC}}$ and the PPC decay time-constant can differ by up to $100 \%$. The contact resistivity when extracted from two contact pairs, selected due to having similar $G_{\mathrm{PPC}}$ decay characteristics, was $0.2 \Omega \mathrm{mm}$ when illuminated and about $0.4 \Omega \mathrm{mm}$ when in dark. Hence, the increase in $r_{\mathrm{c}}$ in dark compared to illuminated is more than $100 \%$. However, the increase has not been statistically verified due the large non-uniformity of the NP sample. Regardless, for this specific case, the practical importance for an HFET is small due to the much larger relative resistance contribution from $R_{\mathrm{sh}}$. For the investigated HFET, the resistance due to material resistivity is about 60-150 $\Omega$ depending on illumination while the resistance due to contacts is only $2-4 \Omega$.

The difference between $R$ and NP suggests that the annealing has increased the surface potential, possibly by formation of a surface oxide as reported by Higashiwaki et $a l{ }^{28}$ The resulting electron distribution with a negligible density in the cap and a significantly reduced density in the channel will result in slower recombination since there is a smaller number of electrons to support the different recombination processes. The large effect of the RS passivation pro- cess may be due to that the reactive sputter deposition process is a plasma based deposition process which contains an Argon plasma cleaning step that may remove the surface oxide. The effect is equivalent to a lowering of the surface potential resulting in a deeper potential minimum in the cap. As a consequence a large density of electrons will be localized inside the cap, allowing for much faster recombination to passivation interface states and barrier states. For $L P$, there is a generally higher electron density and there also exists a significant density of electrons in the cap, providing the means for rapid PPC decay.

Since the three samples have the same epitaxial structure, electron-hole generation in the GaN cap and GaN channel/buffer should have a similar effect for all samples, which is not the case even when compensating for the lower transmittance of the passivating layers. Furthermore, photon energies around the bandgap of $\mathrm{GaN}$ is causing the strongest PPC. Hence, the effect from electron-hole generation in the AlGaN barrier is assumed to be small. This leaves the options of recombination to deep level states and recombination to surface states. A surface state, formed during annealing of non-passivated material, would explain the very large difference between numbers of electrons generated in NP on one hand and $R$ and $L P$ on the other. The activation energy of this trap would then be at least $3.1 \mathrm{eV}$ which is very close to the valence band of $\mathrm{GaN}$. The rough energy resolution of the $G_{\mathrm{PPC}}$ versus photon energy (limited by the use of filters) prevents us from quantifying the ratio of surface states to deep level states.

\section{CONCLUSION}

Even though the HFET device, in its practical applications, most often will be operated in some kind of package, it is the authors' experience that all process control monitoring and most device characterization (at least in research and development environments) is performed with the device somehow illuminated. Failure to take the effects of illumination into account can in a worst case scenario result in erroneous conclusions. An obvious example from this investigation is that the $R_{\mathrm{sh}}$ increase due to the ohmic contact annealing is less than $10 \%$ when illuminated, while the increase is more than $100 \%$ when comparing values measured under dark conditions. Only comparing the illuminated characteristics will not reflect the seriousness of electrical degradation due to annealing.

We propose that the PPC of these materials are mainly caused by the delayed recombination of electrons excited from surface or passivation interface traps and deep level states in the AlGaN. There may also be some small effect of electron-hole generation in GaN and AlGaN. The energy dependence prohibits us to exclude any of these processes. The difference in the time-constant for the PPC decay of the differently processed materials is due to differences in surface physics and resulting electron distribution, where having an electron accumulation layer in the cap is advantageous for rapid PPC decay.

Furthermore, HFETs that are almost completely stable irrespective of illumination conditions have been produced 
by utilizing a LPCVD deposited silicon nitride passivation. The main reason for the lower illumination sensitivity is that a higher sheet density makes the quite small increase of $n_{\mathrm{s}}$ negligible, also the LPCVD silicon nitride is less transparent in the UV-range, blocking about half of the incident photons.

\section{ACKNOWLEDGMENTS}

United Monolithic Semiconductors are acknowledged for supplying the epitaxial material investigated in this report. The Photonics group at the department of Microtechnology and Nanoscience, Chalmers University of Technology, are acknowledged for lending the filters used in this work. Furthermore, this research has been carried out in the Microwave Wide Bandgap Technology project financed by Swedish Governmental Agency of Innovation Systems (VINNOVA), Swedish Energy Agency (STEM), Chalmers University of Technology, Ericsson AB, Furuno Electric Co., Ltd., Infineon AG, Norse Semiconductor Laboratories AB, Norstel AB, NXP Semiconductors BV, and Saab AB.

${ }^{1}$ J. Zhang, X. Hu, A. Lunev, J. Deng, Y. Bilenko, T. M. Katona, M. S. Shur, R. Gaska, and M. A. Khan, Jpn. J. Appl. Phys., Part 1 44, 7250 (2005).

${ }^{2}$ S.-J. Chang, H. Hung, Y.-C. Lin, M.-H. Wu, H. Kuan, and R.-M. Lin, Jpn. J. Appl. Phys., Part 1 46, 2471 (2007).

${ }^{3}$ J. Z. Li, J. Y. Lin, H. X. Jiang, M. A. Khan, and Q. Chen, J. Appl. Phys. 82, 1227 (1997).

${ }^{4}$ X. Z. Dang, C. D. Wang, E. T. Yu, K. S. Boutros, and J. M. Redwing, Appl. Phys. Lett. 72, 2745-2747 (1998).

${ }^{5}$ S. J. Chung, B. Karunagaran, S. Velumani, C. H. Hong, H. J. Lee, and E. K. Suh, Appl. Phys. A: Mater. Sci. Process. A86, 521-524 (2007).

${ }^{6}$ E. Arslan, S. Butun, S. B. Lisesivdin, M. Kasap, S. Ozcelik, and E. Ozbay, J. Appl. Phys. 103, 103701 (2008).

${ }^{7}$ B. K. Li, W. K. Ge, J. N. Wang, and K. J. Chen, Appl. Phys. Lett. 92, 082105 (2008).

${ }^{8}$ P. B. Klein, S. C. Binari, K. Ikossi-Anastasiou, A. E. Wickenden, D. D. Koleske, R. L. Henry, and D. S. Katzer, Electron. Lett. 37, 661 (2001).
${ }^{9}$ T. Mizutani, Y. Ohno, M. Akita, S. Kishimoto, and K. Maezawa, Phys. Status Solidi A 194, 447 (2002).

${ }^{10} \mathrm{P}$. Valizadeh, E. Alekseev, D. Pavlidis, Y. Feng, and H. Morkoc, SolidState Electron. 50, 282 (2006).

${ }^{11}$ C. K. Yang, P. Roblin, A. Malonis, A. Arehart, S. Ringel, C. Poblenz, Y. Pei, J. Speck, and U. Mishra, IEEE MTT-S Int. Microwave Symp. Dig. 1-3, 1209 (2009).

${ }^{12}$ M. Fagerlind, H. Zirath, and N. Rorsman, Semicond. Sci. Technol. 24, 045014 (2009).

${ }^{13}$ J. Y. Shiu, V. Desmaris, N. Rorsman, K. Kumakura, T. Makimoto, H. Zirath, and E. Y. Chang, Semicond. Sci. Technol. 22, 717 (2007).

${ }^{14}$ M. Sudow, M. Fagerlind, M. Thorsell, K. Andersson, N. Billstrom, P. A. Nilsson, and N. Rorsman, IEEE Trans. Microwave Theory Tech. 56, 1827 (2008).

${ }^{15}$ M. Thorsell and K. Andersson, IEEE Trans. Microwave Theory Tech. 60, 149-157 (2012)

${ }^{16}$ K. Shiojima and N. Shigekawa, Jpn. J. Appl. Phys., Part 1 43, 100 (2004).

${ }^{17}$ N. Nepal, J. Li, M. L. Nakarmi, J. Y. Lin, and H. X. Jiang, Appl. Phys. Lett. 87, 242104 (2005).

${ }^{18}$ M. Fagerlind, F. Allerstam, E. O. Sveinbjornsson, N. Rorsman, A. Kakanakova-Georgieva, A. Lundskog, U. Forsberg, and E. Janzen, J. Appl. Phys. 108, 014508 (2010).

${ }^{19}$ J. F. Muth, X. Zhang, A. Cai, D. Fothergill, J. C. Roberts, P. Rajagopal, J. W. Cook, Jr., E. L. Piner, and K. J. Linthicum, Appl. Phys. Lett. 87, 192117 (2005).

${ }^{20}$ M. Motyka, M. Syperek, R. Kudrawiec, J. Misiewicz, M. Rudzinski, P. R. Hageman, and P. K. Larsen, Appl. Phys. Lett. 89, 231912 (2006).

${ }^{21}$ T. E. Cook, Jr., C. C. Fulton, W. J. Mecouch, R. F. Davis, G. Lucovsky, and R. J. Nemanich, Int. J. High Speed Electron. Syst. 14, 107-25 (2004).

${ }^{22}$ C. Nordling and J. Sterman, Physics Handbook, 6 ed. (Studentlitteratur, Lund, 2002).

${ }^{23}$ R. L. Petritz, Phys. Rev. 110, 1254 (1958).

${ }^{24}$ J. Zhao, Z. Lin, T. D. Corrigan, Z. Wang, Z. You, and Z. Wang, Appl. Phys. Lett. 91, 173507 (2007)

${ }^{25}$ R. Gaska, M. Shur, A. Bykhovski, A. Orlov, and G. Snider, Appl. Phys. Lett. 74, 287 (1999).

${ }^{26}$ M. Shur, B. Gelmont, and M. Asif Khan, J. Electron. Mater. 25, 777-785 (1996).

${ }^{27}$ P. Javorka, A. Alam, M. Marso, M. Wolter, A. Fox, M. Heuken, and P. Kordo, Phys. Status Solidi A 194, 472 (2002).

${ }^{28}$ M. Higashiwaki, S. Chowdhury, B. L. Swenson, and U. K. Mishra, Appl. Phys. Lett. 97, 222104 (2010).

${ }^{29}$ S. P. Grabowski, M. Schneider, H. Nienhaus, W. Monch, R. Dimitrov, O. Ambacher, and M. Stutzmann, Appl. Phys. Lett. 78, 2503-2505 (2001). 